\title{
DOSEL DE TRES ESPECIES FORESTALES Y SU RELACIÓN CON LA ADAPTACIÓN A SUELOS DEGRADADOS POR EROSIÓN
}

\section{CANOPY OF THREE FOREST SPECIES AND THEIR RELATION TO ADAPTATION TO BY EROSION DEGRADED SOILS}

\author{
Marco Cabezas Gutiérrez ${ }^{1}$ \\ Fernando Peña Baracaldo ${ }^{2}$ \\ Cristhian Janier Díaz ${ }^{3}$ \\ Andrés Felipe Moreno ${ }^{3}$
}

\section{RESUMEN}

Durante 30 meses, se realizó un estudio en la localidad de Firavitoba, Boyacá, con el objetivo de evaluar y de analizar los cambios en el dosel y la capacidad de adaptación de tres especies forestales de clima frío, Alnus acuminata, Escallonia pendula y Quercus humboldtii, en un suelo degradado por erosión hídrica. Las tres especies fueron sembradas en dos arreglos espaciales, tres bolillos y rectángulo y dentro de esos arreglos, se ubicaron diferentes tratamientos de mezclas simples y combinadas. Se midieron parámetros, como altura de planta, emisión foliar, índice de área foliar, diámetro de la copa y producción de hojarasca. Se observó que $A$. acuminata es una planta de gran sensibilidad al estrés hídrico, al presentar altas pérdidas de material por sequía, mientras que $E$. pendula mostró los mayores índices de sobrevivencia. Sin embargo, A. acuminata es la especie que obtuvo mayores índices de elongación, mayor tasa de emisión foliar y un crecimiento sostenido del IAF, aun cuando presenta periodos de defoliación masiva. Q. humboldtii demostró ser una especie que

1 I.A. M.Sc. cand. Dr. Docente investigador, Facultad de Ingeniería Agronómica U.D.C.A. Dirección para correspondencia: mcabezas@udca.edu.co

2 I.A. M.Sc. Docente investigador U.D.C.A. Dirección para correspondencia fpeña@udca.edu.co.

3 Ingeniero Agrónomo U.D.C.A requiere sombra durante los primeros 24 meses después del transplante, pues al sembrarla individualmente, las plantas crecieron de forma lenta y desarrollaron pocas estructuras foliares. Las tres especies mejoraron las condiciones nutricionales del suelo, debido al aporte de hojarasca. El experimento estableció las condiciones de siembra para las tres especies y concluye quepueden ser usadas, preferiblemente, en mezcla, para programas de reforestación de suelos, con altos niveles de degradación física.

Palabras clave: Área foliar, hojarasca, crecimiento, especies forestales.

\section{SUMMARY}

During 30 months an experiment was carried out at the locality of Firavitoba - Boyacá, to evaluate and analyze the canopy changes and the adaptive capacity of three cold climate forest species, Alnus acuminata H. B. K., Escallonia pendula Pers. and Quercus humboldtii Bompl., in a by water eroded degraded soil. All three species were planted in two spatial arrangements "tres bolillo" and square methods. The species were situated in distinct treatments of simple and compound mixtures. Plant height, leaf emission, leaf area index (LAI), crown diameter expansionaand fallen leaf litter were measured. It was determined that $A$. acuminata is a high sensible plant to drought stress, presenting high losses of planted trees, while, E. pendula showed the major survival index. However, $A$. acuminata had the best elongation index, a 
major leaf rate emission and a constant growth of LAI, even though it showed periods of massive leaf fall. $Q$. humboldtii demonstrated to be a species that needs shading during the first 24 months after transplanting, since, when sown in monoculture, the plant growth was slow and the development of few leaf structures was observed. The three species improved the soil nutrition conditions due to the litter incorporation. The research established the planting conditions for the three species and concluded that it is possible to use them in order to promote reforestation programs, preferably in mixtures, on soils with high erosion levels.

Key words: Leaf area, litter, growth, forest species.

\section{INTRODUCCIÓN}

La idea de mezclar dos o más especies de árboles en una plantación es una práctica normal en la agroforestería, pero no se ha empleado con rigor en explotaciones forestales extensas (Muthuri et al. 2005). Existen ventajas potenciales de las mezclas sobre las siembras en monocultivo, las cuales, se han revisado, ampliamente, en gran variedad de regiones tropicales cálidas, más no en condiciones de altura. Algunas ventajas son la diversificación en la producción de madera, el mayor nivel de diversidad biológica, la reducción de riesgos fitosanitarios, las mejoras en las condiciones del suelo por los aportes de hojarasca y el incremento en los niveles de nutrientes, así como aportes significativos a la sostenibilidad de los ecosistemas (Menalled et al. 1998; Fehse et al. 2002). Las especies de poca tolerancia a la sombra dominan los primeros episodios temporales de la sucesión, mediante altas tasas de asignación de carbohidratos al tallo principal y a las ramas, con el fin de crear copas amplias, capaces de invadir espacio rápidamente (Cermák et al. 2008; Bruijnzeel $\varepsilon$ Veneklaas, 1998). Por el contrario, especies de alta tolerancia a la sombra reparten los fotoasimilados, prevalentemente, a raíces y hojas, conservando copas compactas, en especial en las primeras etapas fenológicas (Oelbermann et al. 2004). Esta condición permite que otras especies las superen en crecimiento, favoreciéndose del sombrío temporal; posteriormente, ajustan sus tasas de elongación y comienzan a crecer con rapidez hasta alcanzar alturas que les permiten dominar las asociaciones vegetales (Moreno $\mathcal{E}$ Cabrera, 2008). El establecimiento en monocultivos ha dominado el manejo intensivo de bosques, tanto en el trópico húmedo, como en las sabanas tropicales, de todo el continente americano (Stape et al. 2008; Pérez-Deveza et al. 2007).

La estimación del índice de área foliar (IAF) es esencial para determinar y modelar las características ambientales del dosel (Monte et al. 2007). El IAF está asociado con los principales factores de producción vegetal, como la interceptación de radiación solar, la eficiencia de conversión de fotoasimilados, la eficiencia en el uso de agua, la eficiencia en el uso del nitrógeno y la regulación de la temperatura y la humedad relativa dentro de la comunidad de plantas (De F. Coello et al. 2007; Barrios \& Cobo, 2004). Este índice, junto con el tamaño del árbol y la forma de la copa, son los más importantes elementos para el estudio de los bosques y plantaciones forestales (Shively et al. 2004; Nascimento et al. 2007). Según Zhang et al. (2005) las especies forestales adaptadas a condiciones de altura tienen mayor capacidad fotosintética, eficiente carboxilación, contenidos de carbono y nitrógeno mayores que las plantas de selvas y sabanas tropicales.

En Colombia, la reforestación se ha enfocado, principalmente, al uso de unas pocas especies introducidas, plantadas en monocultivo, como pinos, cipreses, acacias y eucaliptus, los cuales, por su rápido crecimiento, alta productividad y relativamente fácil manejo, son los abanderados de los productores de madera (De F. Coelho et al. 2007). Las especies nativas, en su mayoría, tienen bajas tasas de crecimiento, presentan desarrollo heterogéneo, son sensibles a condiciones de estrés en el establecimiento y son de rendimiento tardío, lo cual, ha sido impedimento para realizar campañas masivas de siembra (Menalled et al. 1998). El presente trabajo tuvo como objetivos caracterizar el dosel de Aliso (Alnus accuminata H.B.K), de Magle (Escallonia pendula Pers.) y de Roble (Quercus humboldtii Bomp.) en un suelo degradado del municipio de Firavitoba (Boyacá), con diferentes métodos de plantación, comparar el crecimiento en altura de dichas especies durante los primeros 30 meses de establecimiento bajo diferentes mezclas poblacionales, determinar los cambios IAF y cuantificar la hojarasca producida en cada tratamiento. 


\section{MATERIALES Y MÉTODOS}

Localización. Este estudio, se realizó en el municipio de Firavitoba, Boyacá, a 5³9'50" de latitud Norte, 745'10" longitud occidental, una altura de $2450 \mathrm{msnm}$, una temperatura promedio de $15^{\circ} \mathrm{C}, 725 \mathrm{~mm}$ de precipitación anual con distribución bimodal. El sitio experimental corresponde a una formación agroecológica bosque seco montano (Bsmb). Se ubicó en finca de un agricultor, en la vereda Monjas, a una distancia de $5 \mathrm{~km}$ de la ciudad de Sogamoso (IGAC, 2006). La zona, se caracteriza por presentar altos niveles de erosión de suelo. En la actualidad, la mayoría de predios han sido concesionados para la explotación minera (extracción de caliza para la industria cementera), por dos multinacionales. Se escogió un lote con altos niveles de degradación física de suelos, el cual, se encontraba invadido de Eucalyptus globulus y una poca vegetación nativa, con zanjas superficiales, afloramientos de rocas sedimentarias de tamaño medio y baja presencia de gramíneas como cobertura.

Material vegetal. Se sembraron plantas de nueve meses de edad de $A$. acuminata, E. pendula y $Q$. humboldtii, suministrados por el vivero municipal de Tenjo (Cundinamarca). El material, se homogenizó por características visuales, en cuanto a sanidad, longitud y vigor; se tomaron los datos de referencia, longitud de plantas, número de hojas y área foliar para cada una, para tener un parámetro inicial para las mediciones posteriores. La siembra, se realizó el primer semestre del año 2005, en plena temporada de lluvias, para asegurar el prendimiento de todas las plantas. Inicialmente, se tomaron cinco muestras de suelo, que fueron llevadas al laboratorio Agrilab para su caracterización y análisis (Tabla 1). Con base en los resultados, se realizaron los correctivos nutricionales para darle condiciones ideales a las plantas. Se hizo el trazado en curvas de nivel; posteriormente, se marcaron los sitios de siembra, según cada tratamiento y se procedió a realizar los huecos de $40 * 40 * 40 \mathrm{~cm}$, adicionando $120 \mathrm{~g}$ de fertilizante tipo $\mathrm{N}-\mathrm{P}$ $\mathrm{K} 15-15-15,50 \mathrm{~g}$ de un correctivo que contenía $5 \%$ de fósforo, $45 \%$ de calcio y $100 \mathrm{~g}$ de cal dolomita por cada sitio de siembra. Se adicionaron $500 \mathrm{~g}$ de tamo de cereales descompuesto, como aporte de materia orgánica.

Diseño experimental. Se estableció un diseño básico con parcelas divididas subdivididas, donde la parcela principal estuvo conformada por dos métodos de plantación, a saber: rectángulo, donde se sembraron cuatro hileras de árboles, a una distancia de $3 \mathrm{~m}$ entre sí y $2 \mathrm{~m}$ entre plantas, en un área de $90 \mathrm{~m}^{2}$; tres bolillos, con distancia entre planta y planta de $2,5 \mathrm{~m}$ también en $90 \mathrm{~m}^{2}$. La sub-parcela constó de diferentes arreglos de siembra para las tres especies, así: Aliso solo, Magle solo y Roble solo, combinaciones simples en relación 1:1 de las tres especies y una combinación compuesta con igual proporción de individuos de las tres especies. Se empleó un total de 15 tratamientos, con la inclusión de una parcela control, sin siembra alguna de especies, replicados tres veces, para un total de 45 unidades experimentales, en un área efectiva de $4050 \mathrm{~m}^{2}$.

\section{Variables evaluadas}

Crecimiento de las especies forestales. Durante 30 meses, se realizó un seguimiento al crecimiento de las plantas, a partir de los siguientes indicadores: se tomó una altura de referencia inicial por planta en el momento de la siembra; de la unidad experimental, se escogieron cuatro plantas al azar, registrando cada tres meses, cambios en la longitud del tallo principal, la cantidad de ramas producidas y el tamaño de la copa. También, se contó el número de hojas presentes en cada momento de evaluación.

Tabla 1. Resultado del análisis del suelo en el municipio de Firavitoba, finca las Peñitas, al inicio del experimento, promedio de cuatro muestras.

\begin{tabular}{|c|c|c|c|c|c|c|c|c|}
\hline Textura & $\mathbf{p H}$ & Potasio & Calcio & Magnesio & Fósforo & Azufre & C.E & C.0 \\
\hline & & $\mathrm{me} / 100 \mathrm{~g}$ & $\mathrm{me} / 100 \mathrm{~g}$ & $\mathrm{me} / 100 \mathrm{~g}$ & $\mathrm{ppm}$ & $\mathrm{ppm}$ & $\mathrm{dS} / \mathrm{m}$ & $\%$ \\
\hline $\begin{array}{c}\text { Franco } \\
\text { arenoso }\end{array}$ & 5,7 & 0,42 & 6,20 & 1,44 & 6 & 7 & 0,25 & 3,19 \\
\hline
\end{tabular}


Dinámica de la foliación. Con frecuencia trimestral, se contó el número de hojas de las cuatro plantas usadas, como unidad efectiva de evaluación y, con el dato obtenido de laboratorio, se estimó el área foliar por planta.

Índice de área foliar (IAF). El área foliar individual, se midió con un determinador electrónico de scanner marca CID, Inc. Portable Leaf Área Meter CI-202, en 100 hojas tomadas al azar por cada planta. Complementario a lo anterior, se calculó el diámetro de expansión de copa proyectado en el suelo, en cuatro puntos de la circunferencia, utilizando una plomada colocada en la parte terminal de las ramas de mayor expansión, se promedió el dato y se estimó el área de la circunferencia proyectada. Con el dato del área foliar por planta, se procedió a calcular el IAF instantáneo, en cada trimestre, mediante la siguiente fórmula: $\mathrm{IAF}=\mathrm{A}$ foliar por planta $(\mathrm{AF}) / \mathrm{A}$ rea de proyección de copa(AS).

Producción de hojarasca. Siguiendo la metodología propuesta por Frank \& Eduardo (2003), en la parte central de cada unidad experimental se colocaron cajas de madera de $4 \mathrm{~m}^{2}$ y $20 \mathrm{~cm}$ de profundidad, para captar las hojas y los residuos vegetales producidos por las plantas, se colocó una cajuela en una área no sembrada, como control absoluto. Las cajas, se fijaron al suelo para evitar la pérdida de material vegetal por arrastre, dentro de la cajuela. A los 30 meses después de la siembra, se recogieron estos materiales y se pesaron en fresco; luego fueron llevados al laboratorio de suelos de la Universidad de Ciencias Aplicadas y Ambientales, y se sometieron a secado por 72 horas a $65^{\circ} \mathrm{C}$ en una estufa marca Boekel Industries, Inc Model 107905.

Análisis estadístico. Con los datos obtenidos en cada una de las variables evaluadas, se procedió a realizar un análisis de varianza convencional (Martínez E Martínez, 1997), para cada uno de los momentos de evaluación. Una prueba de comparación Duncan fue empleada para estimar la comparación de medias. El análisis, se realizó mediante el programa SAS 2007, en la oficina de apoyo estadístico de la U.D.C.A.

\section{RESULTADOS Y DISCUSIÓN}

Altura de las plantas. Los datos indican que para los primeros 30 meses de crecimiento de los árboles de estas especies, el hecho de escoger un sistema de plantación, tres bolillo o cuadrado es independiente de la respuesta al incremento en altura. La tendencia de crecimiento fue lineal positiva en los tres casos. Existe un mejor comportamiento promedio para $A$. acuminata desde el inicio del ensayo y hasta el final del mismo, seguido por $E$. pendula y por $Q$. humboldtii. Las diferencias en el crecimiento a partir del noveno mes son más evidentes, ya que $A$. acuminata presenta una ganancia en altura por cada periodo de muestreo de casi el doble, comparado a la ganancia de altura de $E$. pendula y es aun mayor con Q. humboldtii (Tabla 2).

Tabla 2. Ganancia de altura de los árboles de Alnus acuminata, Escallonia pendula y Quercus humboldtii durante los primeros 30 meses de plantación en un suelo degrado de Firavitoba, Boyacá.

\begin{tabular}{|c|c|c|c|c|c|}
\hline Tiempo & Q. humboldtii & E. pendula & A. acuminata & $\mathbf{R}^{2}$ & C.V \\
\hline 3 meses & $3,17 \mathrm{~b}$ & $17,90 \mathrm{a}$ & $15,58 \mathrm{a}$ & 0,93 & 19,31 \\
\hline 6 meses & $4,88 \mathrm{~b}$ & $21,67 \mathrm{a}$ & $17,31 \mathrm{a}$ & 0,91 & 16,66 \\
\hline 9 meses & $5,95 \mathrm{~b}$ & $15,39 \mathrm{a}$ & $19,40 \mathrm{a}$ & 0,90 & 17,55 \\
\hline 12 meses & $3,88 \mathrm{~b}$ & $9,85 \mathrm{~b}$ & $21,93 \mathrm{a}$ & 0,94 & 15,0 \\
\hline 15 meses & $5,37 \mathrm{~b}$ & $9,33 \mathrm{~b}$ & $16,56 \mathrm{a}$ & 0,92 & 16,61 \\
\hline 18 meses & $4,82 \mathrm{~b}$ & $8,43 \mathrm{ab}$ & $15,50 \mathrm{a}$ & 0,93 & 15,75 \\
\hline 21 meses & $6,36 \mathrm{~b}$ & $9,38 \mathrm{ab}$ & $13,75 \mathrm{a}$ & 0,91 & 18,03 \\
\hline 24 meses & $5,23 \mathrm{~b}$ & $11,20 \mathrm{a}$ & $13,56 \mathrm{a}$ & 0,94 & 14,08 \\
\hline 27 meses & $8,59 \mathrm{ab}$ & $7,86 \mathrm{~b}$ & $13,06 \mathrm{a}$ & 0,94 & 15,45 \\
\hline 30 meses & $3,74 \mathrm{~b}$ & $8,40 \mathrm{ab}$ & $11,57 \mathrm{a}$ & 0,93 & 15,33 \\
\hline
\end{tabular}

Promedios de tres repeticiones y dos sistemas de plantación. Valores seguidos de la misma letra no presentan diferencias significativas, según la prueba de Duncan $(P \leq 0,05)$. 
La alta tasa de elongación de $A$. acuminata, se debió, posiblemente, a la facilidad en la captación de nitrógeno atmosférico, lo que contribuye con la optimización del carbono (Murcia, 1997). Una mejor asignación de nitrógeno genera una mayor producción de Rubisco, mejorando la eficiencia fotosintética; a su vez, contribuye con una mejor distribución de los carbohidratos tal y como lo proponen Benson E Sylvester (1993). También corrobora estudios que muestran la capacidad de crecimiento de especies fijadoras de nitrógeno atmosférico en suelos degradados (Franco $\mathcal{E}$ Faria, 1997; Chada et al. 2004; Baliero et al. 2007).

Para el caso de E. pendula, se observa que el mejor tratamiento, especialmente, a partir de la mitad de duración del experimento fue la mezcla de dicha especie con Q. humboldtii. E pendula es una planta históricamente presente en la zona y se podría decir que tiene una ventaja comparativa con las otras dos especies, razón por la cual se esperaba un comportamiento mejor en cuanto a crecimiento se refiere. Es posible que la menor tasa de elongación en comparación con aliso, se deba a una estrategia para evitar los daños por la deficiencia de agua. Esta aseveración está sustentada en las ideas propuestas por Do et al. (2005) y Stape et al. (2008), quienes exponen un planteamiento similar para especies, como Acacia tortilis y Eucaliptus grandis, E. urophylla en condiciones de déficit hídrico en los trópicos.

Respecto a Q. humboldtii, en el caso de la siembra monoespecífica, las plantas tuvieron serias limitaciones para la elongación de los tallos, se notó alta suceptibilidad a la luz directa y tendencia la ramificación lateral corta. Cuando las plantas se sembraron en mezcla con las otras especies produjeron entrenudos más largos, menor ramificación lateral, producto de la dominancia apical. Es importante destacar que $Q$ humboldtii forma parte de sucesiones secundarias, normalmente, asociado en sus primeros años de vida a arbustos y plantas de sotobosque que le brindan protección de la radiación directa, le proveen sombra a sus estructuras y le permiten un establecimiento apropiado hasta que la planta tenga la suficiente capacidad de alcanzar altura y dominar el dosel de las asociaciones vegetales, en concordancia con lo expuesto por López-Barrera \& Newton (2006). Las observaciones encontradas en el presente trabajo son sustentadas por los postulados de Menalled et al. (1998), sobre el comportamiento de otras especies tropicales, como Cedrella odorata, Cordia alliodora y Hyeronima alchorneoides y por lo expuesto por Cermák et al. (2008), en dos especies de Quercus, en condiciones de clima mediterráneo.

Dinámica de la foliación en cada especie. A. acuminata presentó temporadas de alta emisión seguidas por temporadas de alta defoliación, lo cual, confirma su característica semicaduca (Segura et al. 2006). Los periodos de defoliación, se produjeron al final de las estaciones secas, mientras que las lluvias indujeron alta renovación de follaje, hecho que confirma la dependencia de la especie al hidroperiodo. La defoliación y la nueva emisión de hojas, se presentaron en toda la rama; en los terminales permanecieron hojas fotosintéticamente activas. E. pendula siempre conservó una tasa constante de emisión foliar, la cual, se incrementó conforme al crecer el número de ramas laterales; las hojas, constantemente permanecieron verdes en los terminales de las ramas, mientras que en la parte proximal de las mismas, rápidamente se presentaba un cambio de coloración de verde a violeta, lo cual, fue un indicio de la senescencia foliar y la posterior caída. Q. humboldtii mostró una tendencia constante al incremento en el número de hojas y aunque renovó follaje, permanentemente, el desprendimiento masivo de hojas no fue tan evidente, como el caso de las otras dos especies. En la figura 1, se puede observar el comportamiento de cada una de las especies en cuanto al número de hojas en cada periodo de evaluación. Se puede afirmar que bajo las condiciones del experimento, Q. humboldtii se comportó como una planta de follaje permanente, con emisiones foliares reguladas por las temporadas de lluvia.

A partir de los 15 meses iniciales del ensayo hasta el final, A. acuminata presentó mayor número de hojas, posiblemente, debido al incremento en la altura de la planta, lo cual, de forma directa, conlleva a un mayor número de ramas. Q. humboldtii, de acuerdo al expuesto por Ramírez et al. (2003) presenta un mejor desarrollo si durante los primeros meses es favorecido con sombra, postulado que confirma lo expresado en la presente investigación. Aspectos similares son propuestos y destacados por Moreno \& Cabrera (2008) en Q. ilex, la cual, es proclive a crecer en asociaciones de plantas que le provean sombra en los primeros años de crecimiento. Q. humboldtii sembrada en condiciones de monocultivo muestra los menores valores de hojas producidas, pero 


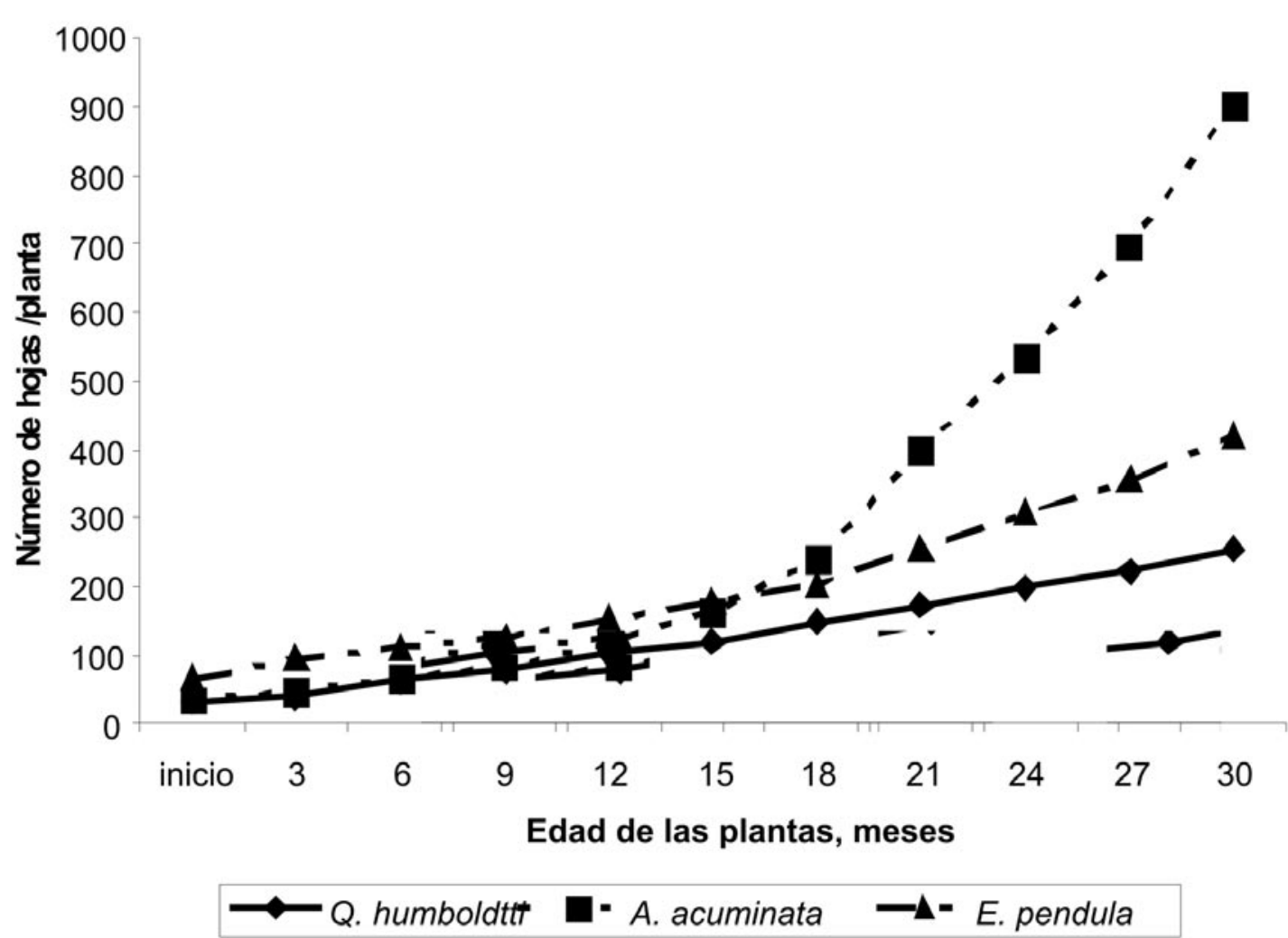

Figura 1. Número de hojas desplegadas presentes en cada periodo de evaluación, de Alnus acuminata, Escalonia pendula y Quercus humboldtii, durante los primeros 30 meses después de la siembra en un suelo erosionado.

también las menores tasas de elongación del tallo. Estas condiciones hacen que la planta tenga aspecto achaparrado, talla pequeña, con copa cerrada, razones esenciales a la hora de interceptar y aprovechar la radiación fotosintéticamente activa (RFA).

Índice de área foliar (IAF). En las figuras 2A, 2B y 2C, se muestra el comportamiento en el tiempo del IAF para cada especie, lo cual, explica la naturaleza de ellas respecto a la estructura del dosel. El IAF de $A$. acuminata mostró una tendencia de tipo polinómico durante el tiempo, producto de la defoliación parcial de la planta durante los periodos de sequía. El aliso es una planta que tiene copa piramidal, con producción de ramas plagiotrópicas primarias y secundarias, renovación rápida de follaje y basa su arquitectura en un eje central recto y fuerte. Esta característica permite que el IAF esté en cambio permanente, con tendencia positiva, diferente a las especies netamente caducas; algunas de ellas bien descritas por Menalled et al. (1998). El coeficiente de regresión lineal $(0,72)$ fue inferior al polinómico $(0,92)$, por ese motivo, se asume que $A$. acuminata no tiene tendencia lineal positiva en el IAF durante los primeros 30 meses de crecimiento.

$E$. pendula fue la especie que mostró un IAF mayor en los primeros meses después de la siembra, debido a que posee mayor área foliar por hoja en promedio (Figura 1) y la expansión de copa es baja. El IAF no cambia en forma definida durante los primeros 30 meses de crecimiento de la especie y se debe a que la planta tiene un hábito de foliación bien establecido hacia la parte terminal de las ramas y una buena proporción de ellas queda desnuda, por tanto, aun cuando la planta crezca en diámetro de copa, no lo hará de la misma forma en área foliar. Las ramas del magle en su mayoría son plagiotrópicas, pero también se presenta una buena cantidad de tallos prolépticos de tipo ortotrópico hacia el interior de la planta, lo que no permite modelar, 

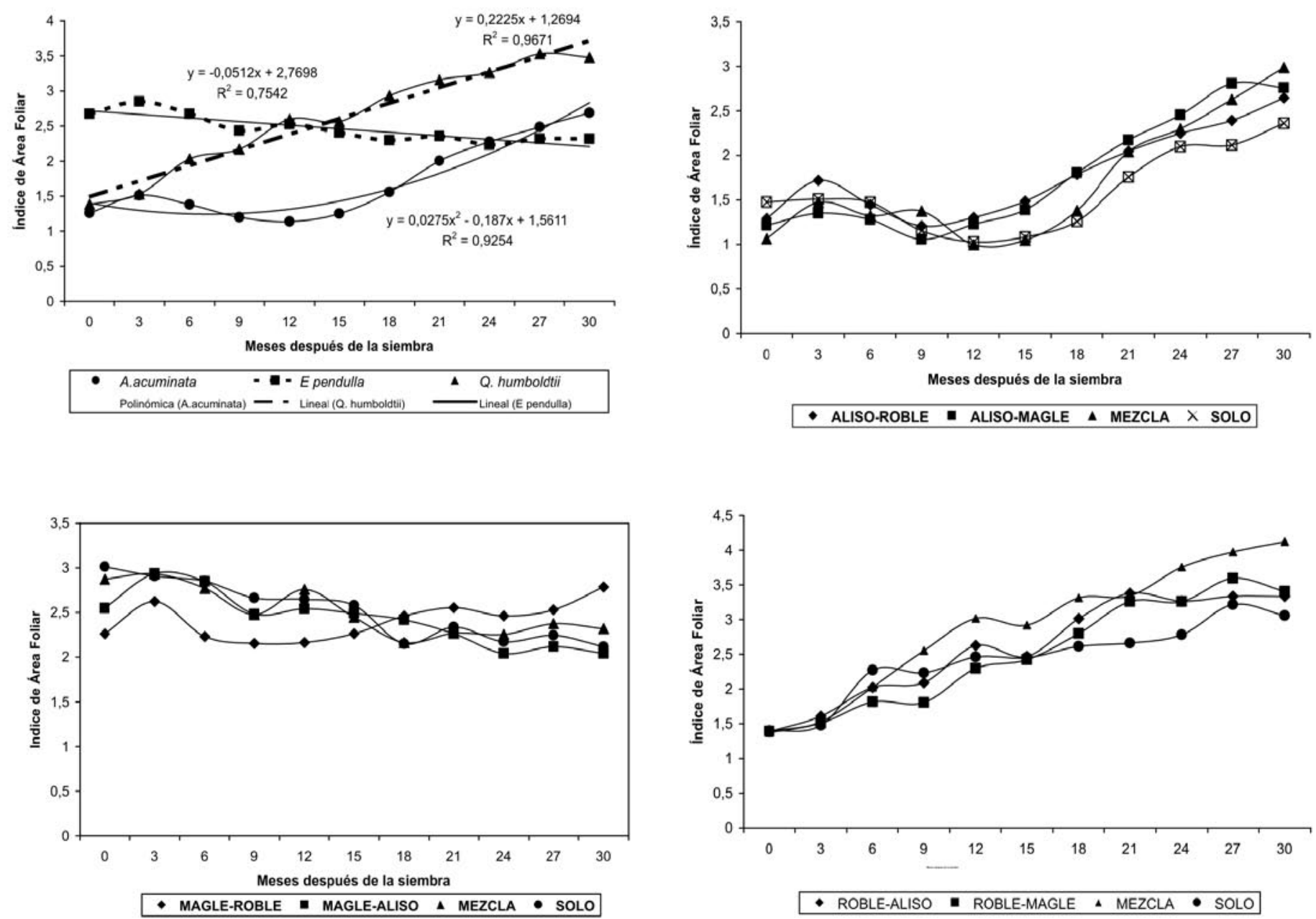

Figura 2. Índice de Área Foliar (IAF) instantáneo promedio de Alnus acuminata, Escallonia pendula y Quercus humboldtii durante los primeros 30 meses después de la siembra en un suelo erosionado. Diferentes tipos de mezclas. A Tendencia general del IAF para cada especie. B. Comportamiento del IAF en A. acuminata solo y en mezcla. C. comportamiento de E. pendula, solo y en mezcla. D, comportamiento de $Q$. humboldtii solo y en mezcla.

fácilmente, la estructura de esta planta. La falta de estudios sobre adaptación y comportamiento de esta especie, no permitieron hacer comparaciones con los datos encontrados en el presente trabajo.

Con el transcurso del tiempo, se notó un incremento lineal positivo en $Q$. humboldtii, con aumento regulado en el tiempo. El roble fue la especie que menor tasa de emisión de hojas presentó, pero las hojas y las cortas ramas formaron copas compactas, las plantas tuvieron ramificación lateral restringida y eso provocó que el área de expansión de la copa fuera baja. Es posible que esta planta trate de aprovechar al máximo la radiación fotosintéticamente activa (RFA), para producir carbohidratos que se transloquen en forma mayoritaria a la raíz, en vez de ser asignados a las ramas, tal y como lo afirman Pérez-Deveza et al. (2007), quienes hacen observaciones similares en $Q$. suber y Cermák et al. (2008), con resultados parecidos en $Q$. pubescens y $Q$. ceris.

El coeficiente de regresión lineal para la variable fue superior al $96 \%$, lo cual, asegura un comportamiento 
lineal del IAF para Q. humboldtii. Para ninguna de las especies, se logró obtener el IAF máximo, pues en ningún caso se cerraron surcos de siembra. El valor mayor de IAF en $Q$. humboldtii se logró al final del ensayo, como era de esperarse, con una cifra de 3 , 5, inferior a la reportada por Cermák et al. (2008) y Drunasky \& Struve (2005), quienes obtuvieron valores de IAF entre 5 y 7 para otras especies de roble.

Al observar la dinámica del IAF en $A$. acuminata sembrado en diferentes mezclas con las otras dos especies, figura $2 \mathrm{~B}$, se encuentra que no existen diferencias significativas entre los cuatro tratamientos comparados para ninguna época del ensayo. La tendencia en todos los caso fue similar y muy cercana al promedio general de la especie. En el caso de $E$. pendula, figura $2 \mathrm{C}$, tres tratamientos tuvieron tendencia ligeramente decreciente pero lineal y solamente uno, la mezcla con $Q$. humboldtii, tuvo comportamiento aleatorio, independiente de la edad de la planta. El roble por su baja tasa de elongación y lento desarrollo de copa, no ejerce competencia sobre el magle, lo que sí hace directamente el aliso. Por tal motivo, los tratamientos que contiene esta especie hacen que las planta de magle tenga ramas más largas, las cuales, concentran las hojas en la parte terminal, pero hacen que el diámetro de expansión de copa esté en incremento permanente y, por tanto, se disminuya el valor de IAF, tal y como se observa en la figura 2.

E. pendula parece ser la especie más sensible a la sombra; regula su IAF produciendo un buen número de ramas para captar e invadir espacio en el menor tiempo posible y así no sufrir por estrés lumínico, mientras que Q. humboltii incrementa el IAF sin tener que elongar sus ramas ni el tallo principal, sino mediante la conformación de una copa muy densa y compacta, capaz de absorber la RFA; esta radiación es en mayoría difusa, al estar bajo efectos de la sombra. Según Zhang et al. (2005), las especies forestales adaptadas a condiciones de altura tienen mayor capacidad fotosintética, eficiente carboxilación, contenidos de carbono y nitrógeno mayores que las plantas de selvas y sabanas tropicales. Sin embargo, se debe considerar que las especies leñosas no maximizan las tasas fotosintéticas, aun cuando las condiciones de crecimiento les sean beneficiosas (Warren E Adams, 2004), más bien regulan el proceso para disminuir, en parte, las tasas de fotorespiración y utilizar el nitrógeno en construcción de proteínas distintas a Rubisco. Si es así, A. acuminata será, incluso la especie de menor eficiencia de conversión, pues los datos encontrados por Tangarife (2005) fueron de 2,6 $\mu \mathrm{mol}$ de $\mathrm{CO} 2 \mathrm{~m}^{-2} \mathrm{~s}^{-1}$, comparado con datos de $Q$. pannosa, una especie cultivada en altura en China, la cual, tiene una fotosíntesis neta superior a $8 \mathrm{umol} \cdot \mathrm{m}^{-2}$ $\cdot \mathrm{s}^{-1}$ (Zhang et al. 2005). No se encontraron datos para E. pendula en la literatura científica.

Producción de hojarasca. El promedio de caída de hojarasca para los treinta meses para $A$. acuminata, $E$. pendula y $Q$. humboldtii fue $364,31,359,05$ y $126,90 \mathrm{~kg}$ por parcela de $4 \mathrm{~m}^{2}$, respectivamente, siendo la fracción foliar la que mayor participación tuvo en la producción total. La hojarasca encontrada y recogida no tuvo componentes medianos ni gruesos (Ramírez et al. 2003), solamente contenía material fin y mantillo. Los mejores valores durante esta investigación los obtuvieron $A$. acuminata y $E$. pendula (Figura 3 ), con sus respectivas combinaciones, sin registrar gran diferencia entre estas dos especies, $A$. acuminata presenta gran producción de hojas y un comportamiento que es característico de una especie semi-caduca, lo que representa un mayor aporte de biomasa. Además, el aporte de hojarasca es utilizado por la planta en el momento que se descompone, aprovechando altos contenidos de nitrógeno que sirve y que es necesario en la composición de proteínas, ácidos nucleicos y otros componentes celulares, siendo así una molécula esencial para el crecimiento de todos los organismos.

Al contrario, E. pendula presentó una menor producción de hojas, pero un alto índice de área foliar individual, aportando un volumen similar al obtenido por $A$. acuminata. Estas dos especies originaron una mayor cantidad de materia orgánica en forma de cobertura evitando la entrada directa del sol, lo que a su vez se ve reflejado en una mayor humedad para el desarrollo de microorganismos descomponedores de materia orgánica. Q. humboltii, presentó la más baja producción de biomasa, aspecto que sumado a lo obtenido en las otras variables, permite definir que su comportamiento no fue favorable, debido a la baja capacidad de adaptación a condiciones secas y suelos erosionados. Al obtener la suficiente altura y tamaño de copa, el reciclaje de nutrientes, producto de la caída de hojas, acondiciona un nicho especial para su supervivencia, permitiendo que dicha especie adquiera mejor adaptabilidad. Las fluctuaciones estacionales en la producción de hojarasca están reguladas, fundamentalmente, por procesos y 


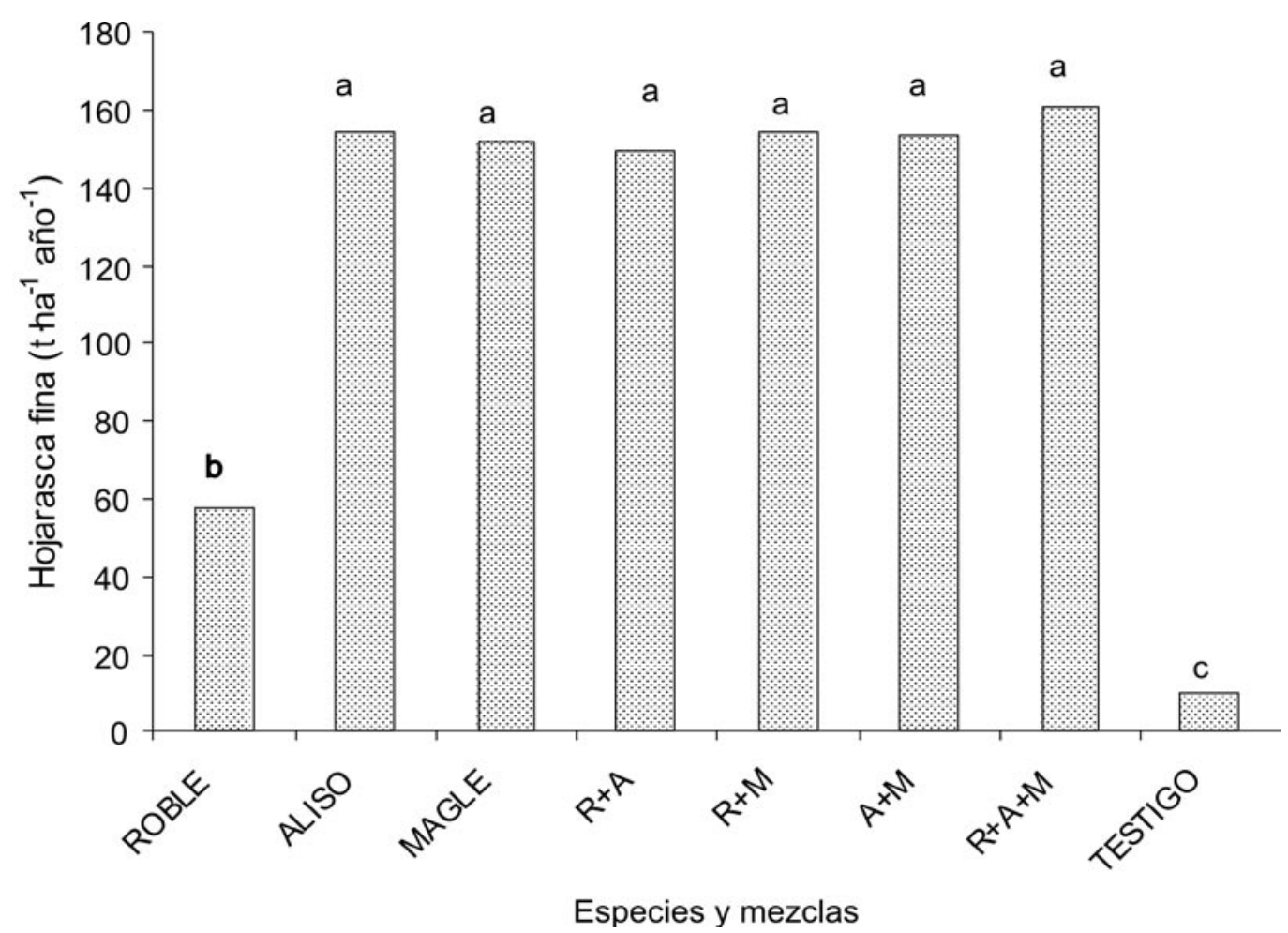

Figura 3. Estimación de la producción de hojarasca y mantillo en las parcelas de siembra de Alnus acuminata, Escallonia pendula y Quercus humboldtii en un suelo degradado en Firavitoba-Boyacá. Barras con la misma letra no presentan diferencias significativas, según la prueba de Duncan $(P \leq 0,05)$.

por factores biológicos y climáticos, aunque también son relevantes la topografía, las condiciones edáficas, la especie vegetal y la edad de la especie (Brown, 1997). Estudios en bosques brasileños demostraron que especies deciduas y semideciduas presentan mayor cantidad de hojas al final de la estación seca, lo que demuestra estrecha relación entre decidualidad y estacionalidad climática (Morellato, 1995; Vital et al. 2004). Para el presente experimento, en todos los casos, los valores de hojarasca producida son menores a los reportados por Vargas-Parra E Varela (2007), en bosques andinos de altura, los cuales, oscilaron al rededor de $8 \mathrm{mg} \cdot \mathrm{ha}^{-1} \cdot$ año.

\section{CONCLUSIONES}

Bajo las condiciones del presente trabajo, el tipo de siembra de las tres especies evaluadas no afectó su desarrollo. Se puede recomendar la siembra en tres bolillo, ya que ahorra espacio y permite tener un mayor número de plantas por área. Se demuestra que $Q$. humboldtii es una especie susceptible a la siembra en monocultivo, siendo necesario plantar en forma intercalada, una especie de mayor tasa de crecimiento para favorecer la producción rápida de sombra y así asegurar el prendimiento y la sobrevivencia de la plantación.

Las tres especies evaluadas pueden hacer parte de un programa mixto de reforestación para la zona en estudio. De esta manera, se contribuye a la diversificación productiva y muy posiblemente, al equilibrio biológico. Ninguna de las especies llegó al IAF máximo, debido, a las tasas de crecimiento lento en el trópico alto. Se sugiere seguir tomando datos para encontrar estos indicadores.

En todos los tratamientos hubo aporte substancial de hojarasca al suelo, en comparación con la porción no intervenida del predio. Queda la expectativa sobre la producción a futuro de hojarasca y el aporte de ramas gruesas y otros componentes estructurales, cuando las plantas alcancen mayor altura y las copas logren su plena formación. 


\section{BIBLIOGRAFÍA}

BALIEIRO, F.C.; FRANCO, A.A.; FONTES, R.L.F.; DIAS, L.E.; FARIA, S.M. 2007. Evaluation of the throughfall and stemflow nutrient contents in mixed and pure plantations of Acacia mangium, Pseudosamenea guachapele and Eucalyptus grandis. Rev. Árvore 31(2):339-346.

BARRIOS, E.; COBO, J.G. 2004. Plant growth, biomass production and nutrient accumulation by slash/agroforestry systems in tropical hillsides of Colombia. Agrofor. Syst. 40(3):255-265.

BENSON, D.R.; SYLVESTER, W.B. 1993. Biology of Frankia strains, actinomycete symbionts of actinorhizal plants. Microbiol. Rev. 57:293-319.

BROWN, S. 1997. Estimating biomass and biomass change of tropical forests. A primer. FAO Forestry Paper 134. Roma, 55p.

BRUIJNZEEL, L.; VENEKLAAS, E. 1998. Climatic conditions and tropical montane forest productivity: the fog has not lifted yet. Ecology. 79(1):3-9.

CERMÁK, J.; TOGNETTI, R.; NADEZHDINA, N.; RASCHI, A. 2008. Stand structure and foliage distribution in Quercus pubescens, and Quercus ceris forest in Tuscany (Central Italy). For. Ecol. Managem. 254:118-128.

CHADA, S.S.; CAMPELLO, E.F.C.; FARIA, S.M. 2004. Natural succession under a nitrogen-fixing legume trees stand in a hillside at Angra dos Reis - RJ, Brazil. Rev. Árvore 28(6):801-809.

De F. COELLO, S.R.; De M. GONCALVES, J.I.; De MIRANDA, M.S.L.; MOREIRA, M.R.; DA SILVA, E.V.; LACLAUD, J. P. 2007. Crescimento, nutrição et fixação biológica de nitroggenio em plantios mistos de eucalipto e leguminosas arbóreas. Pesq. Agrop. Bras. 42(6):759-768.

DO, F.C.; GOUDIABY, V.A.; GIMENES, O.; DIAGNE, A. L.; DIOUF, M. 2005. Environmental influence on canopy phenology in the dry tropics. Forest Ecol. Managem. 215:319-328.
DRUNASKY, N.; STRUVE, D.K. 2005. Quercus macrocarpa and $Q$. prinus physiological responses to drought stress and their potencial for urban forestry. Urban For. E Urban Greed. 4:13-22.

FEHSE J.; HOFSTEDE R.; AGUIRRE N.; PALADINES CH.; KOOIJMAN, A.; SEVINK J. 2002. High altitude tropical secondary forest: a competitive carbon sink? For. Ecol. Manage. 163:9-25.

FRANCO, A.A.; FARIA, S.M. 1997. The contribution of $\mathrm{N}_{2}$-fixing tree legumes to land reclamation and sustainability in the tropics. Soil Biol. and Bioch. 29(5/6):897-903.

FRANK, B.; EDUARDO, S. 2003. Biomasa dinamics of Erytrina lanceolada as influenced by shootpruning intensity in Costa Rica. Agrofor. Syst. 57(1):19-28.

INSTITUTO GEOGRÁFICO AGUSTIN CODAZZI. 2006. Estudio general de suelos de los municipios de Aquitania, Cuitiva, Firavitoba, Iza, Mongui, Nobsa Sogamoso, Tibasosa, Tópaga y Tota departamento de Boyacá. Subdirección Agrológica Bogotá.

LÓPEZ-BARRERA, F.; NEWTON, A. 2006. Edge type effect on germination of oak tree species in the Highlands of Chiapas Mexico. Forest. Ecol. Managem. 223:327-335.

MARTÍNEZ, L.N.; MARTÍNEZ B.R. 1997. Diseño Experimental. Análisis de datos estándar y no estándar. Bogotá, Unilibros. 640p.

MENALLED, F.D.; KELTY, M.J.; EWEL, J.J. 1998. Canopy development in tropical three plantations: a comparison of species mixtures and monocultures. Forest. Ecol. Managem. 104:249-263.

MORELLATO, L.P.C. 1995. As estações do ano na floresta. In: Morellato, L.P.C.; Leitão Filho H.F. (Eds.) Ecologia e preservação de uma floresta tropical urbana: Reserva de Santa Genebra. Campinas: Editora da Unicamp, p.37-41.

MONTE, M.A.; DAS, G.F.M.; REIS, G.G.; DOS REIS, H.G., LEITE, H.G.; STOCKS, J.J. 2007. Métodos 
inderetos to estimação da cobertura de dossel em povoamentos de clone de eucalipto. Pesq. Agrop. Bras. 42(6):760-769.

MORENO, G.; CABRERA, E. 2008. Impact of stand density on water status and leaf gas exchange in Quercus ilex. Forest Ecol. Managem. 254:74-84.

MURCIA, C. 1997. Evaluation of Andean alder as a catalyst for the recovery of tropical cloud forest in Colombia. Forest. Ecol. Managem. 99(1,2):163170.

MUTHURI, C. W.; ONG C. R.; NGUMI V. W.; MATI B. M. 2005. Tree and crop productivity in Grevillea, Alnus and Pawlonia-based agroforestry systems in semi- arid Kenya. Forest Ecol. Managem. 212:23-39.

NASCIMENTO, A.R.T.; FAGG, J.M.F.; FAGG, C.W. 2007. Canopy openness and lai estimates in two seasonally deciduous forests on limestone outcrops in central Brazil using hemispherical photographs. Rev. Árvore 31(1):167-176.

OELBERMANN, M.; VORONEY R.P.; GORDON A.M. 2004. Carbon secuestration in tropical and temperature agroforestry systems: a review with examples from Costa Rica and Southern Canada. Agric. Ecosyst. E Environm. 104:359-377.

PÉREZ-DEVEZA, M.; CORTINA, J.; VILAGROSA, A.; VALLEJO, R. 2007. Shrubland management to promote Quercus suber L. establischment. Forest Ecol. Managem. 253:118-128.

RAMÍREZ, J.A.; ZAPATA, C.M.; LEÓN, J.D. 2003. Descomposición de hojarasca en bosques montanos naturales de Quercus humboldtii y reforestados (Pinus patula y Cupressus lusitánica) de la región de Piedras Blancas, Antioquia. 1er Simposio Forestal DCF, Medellín: U. Nacional de Colombia, p.29-35.
SEGURA, M.; KANNINEN, M.; SUÁREZ, D. 2006. Allometric models for estimating aboveground biomass of shade trees and coffeee bushes growth together. Agrofor. Syst. 68:143-150.

SHIVELY, G.E.; ZELEK, C.; MIDMORE, D.J.; NISSEN, T.M. 2004. Carbon secuestration in a tropical landscape: an economic model to medition it incremental cost. Agrofor. Syst. 60(3):189-197.

STAPE, J.L.; BINKLEY, D.; RYAN, M.G. 2008. Production and carbon allocation in a clonal Eucalyptus plantation with water and nutrient manipulations. Forest. Ecol. Managem. 255:920-930.

TANGARIFE, P.G. 2005. Validación de dos modelos matemáticos relacionados con la estimación de acumulación de biomasa en las especies forestales Alnus acuminata H.B.K, Eucalyptus grandis y Eucalyptus pellita. Trabajo de grado Ingeniero Agrónomo, Universidad de Caldas. 84p.

VARGAS-PARRA, L.; VARELA, A. 2007. Producción de hojarasca en un bosque de niebla en la reserva natural La Planada (Nariño- Colombia). Universitas Scientiarum. 12:35-49.

VITAL, A.R.T.; GUERRINI, I.A.; FRANKEN, W.K. 2004. Produção de serapilheira e ciclagem de nutrientes de uma floresta estacional semidecidual em zona ripária. Rev. Árvore. 28(6):793-800.

WARREN, C. R.; ADAMS, M. A. 2004. Evergreen trees do not maximize instantaneous photosynthesis. Trends Plant. Sci. 9(6):270-274.

ZHANG, S.; ZHOU Z.; HU H.; XU K.; YAN K.; LI.S. 2005. Photosynthetic perfomances of Quercus pannosa vary with altitude in the Hengduan Montains, southwest China. Forest Ecol. Managem. 212:291-301.

Recibido: Junio 10 de 2008

Aceptado: Octubre 17 de 2008 